\title{
Generalized Cesàro operators on certain function spaces
}

\author{
by Sunanda NaIK (Bhubaneswar)
}

\begin{abstract}
Motivated by some recent results by Li and Stević, in this paper we prove that a two-parameter family of Cesàro averaging operators $\mathcal{P}^{b, c}$ is bounded on the Dirichlet spaces $\mathcal{D}_{p, a}$. We also give a short and direct proof of boundedness of $\mathcal{P}^{b, c}$ on the Hardy space $H^{p}$ for $1<p<\infty$.
\end{abstract}

1. Introduction and preliminaries. Let $\Delta$ be the unit disc in the complex plane $\mathbb{C}$ and $d m(z)=r d r d \theta / \pi$, the normalized Lebesgue area measure on $\Delta$. Let $\mathcal{H}$ be the space of all analytic functions in $\Delta$. Recall that for $p>0$, the Hardy space $H^{p}$ consists of $f \in \mathcal{H}$ such that

$$
\|f\|_{p}=\lim _{r \rightarrow 1} M_{p}(r, f)<\infty,
$$

where the integral mean $M_{p}(r, f)$ is defined by

$$
M_{p}(r, f)=\left\{\frac{1}{2 \pi} \int_{0}^{2 \pi}\left|f\left(r e^{\theta}\right)\right|^{p} d \theta\right\}^{1 / p}, \quad 0 \leq r<1 .
$$

For $a, p>0, \mathcal{D}_{p, a}$ denotes the space of all $f \in \mathcal{H}$ such that

$$
\|f\|_{\mathcal{D}_{p, a}}^{p}=|f(0)|^{p}+\int_{\Delta}\left|f^{\prime}(z)\right|^{p}(1-|z|)^{a} d m(z)<\infty .
$$

Let $\omega(r), 0 \leq r<1$, be a positive weight function which is integrable on $[0,1)$. We extend $\omega$ on $\Delta$ by setting $\omega(z)=\omega(|z|)$.

For $0<p<\infty$, the weighted Bergman space $\mathcal{B}_{\omega}^{p}$ consists of $f \in \mathcal{H}$ such that

$$
\|f\|_{\omega, p}^{p}=\int_{\Delta}|f(z)|^{p} \omega(z) d m(z)<\infty .
$$

Using the definition of integral mean, the above norm in $B_{\omega}^{p}$ can be written

2010 Mathematics Subject Classification: 30D45, 30D60, 33C05, $47 \mathrm{~B} 38$.

Key words and phrases: hypergeometric functions, generalized Cesàro operators. 
as

$$
\|f\|_{\omega, p}^{p}=2 \int_{0}^{1} M_{p}^{p}(r, f) \omega(r) r d r
$$

Note that $B_{\omega}^{p}$ is a Banach space when $1 \leq p<\infty$ and Hilbert space for $p=2$.

For any complex numbers $a, b, c \neq-n, n=0,1,2, \ldots$, the Gaussian hypergeometric function [AAR, T] is defined by power series expansion

$$
{ }_{2} F_{1}(a, b ; c ; z):=F(a, b ; c ; z)=\sum_{n=0}^{\infty} \frac{(a, n)(b, n)}{(c, n)} \frac{z^{n}}{n !} \quad(|z|<1),
$$

where $(a, n)$ is the shifted factorial defined by Appel's symbol

$$
(a, n):=a(a+1) \ldots(a+n-1)=\frac{\Gamma(a+n)}{\Gamma(a)}, \quad n \in \mathbb{N}=\{1,2, \ldots\},
$$

and $(a, 0)=1$ for $a \neq 0$.

For $f(z)=\sum_{n=0}^{\infty} a_{n} z^{n} \in \mathcal{H}$, the Cesàro operators of type $(1, b ; c)$ or simply the generalized Cesàro operators are defined as

$$
\mathcal{P}^{b, c} f(z):=\sum_{n=0}^{\infty}\left(\frac{1}{A_{n}^{b+1 ; c}} \sum_{k=0}^{n} b_{n-k} a_{k}\right) z^{n}
$$

where

$$
A_{k}^{a, b ; c}=\frac{(a, k)(b, k)}{(c, k)(1, k)}, \quad A_{k}^{b ; c}=\frac{(b, k)}{(c, k)}
$$

and $b_{k}$ is given by $b_{0}=1$, and for $k \geq 1$,

$$
b_{k}=\frac{1+b-c}{c} A_{k-1}^{b+1 ; c+1}=\frac{1+b-c}{b} A_{k}^{b ; c} .
$$

These operators were introduced in AHLNP and have been studied to prove the boundedness on Hardy spaces, BMOA and Bloch space. For $b=\gamma+1$ and $c=1$, we obtain the Cesàro operators of order $\gamma$, or simply the $\gamma$-Cesàro operators $\mathcal{P}^{1+\gamma, 1} f=\mathcal{C}^{\gamma} f(\operatorname{Re} \gamma>-1)$. In particular, for $\gamma=0$, we obtain the classical Cesàro operator $\mathcal{P}^{1,1} f=\mathcal{C} f$. Many authors, for example [M], Si1] and [Si2], have studied the boundedness of $\mathcal{C}$ on $H^{p}, 0<p<\infty$, and the same problem for the Bergman space has been studied by [Si3]. Also in G] the boundedness of $\mathcal{C}$ on the Dirichlet space $\mathcal{D}_{p, a}$ has been proved when $p=2$ and $a>0$. For any $\gamma \in \mathbb{C}$ with $\operatorname{Re} \gamma>-1$, the operators $\mathcal{C}^{\gamma}$ were introduced in [St] and proved to be bounded on Hardy space. Subsequently the boundedness of $\mathcal{C}^{\gamma}$ has been studied by Xia $[\underline{\mathrm{X}}]$ on $H^{p}$ spaces, BMOA and Bloch space, and Stević [S1] proved their boundedness on Dirichlet space. In this article we generalize Stević's results by proving the boundedness of the operator $\mathcal{P}^{b, c}$ on Dirichlet space $\mathcal{D}_{p, a}$ for $p>1$, and using this result we prove its boundedness on weighted Bergman space $B_{\omega}^{p}$. For some extension in the case of the unit polydisk $\Delta^{n}$ we refer to [CS1, CS2], [CLS] and [S2]. 
In AHLNP] the authors have proved that the operator $\mathcal{P}^{b, c}$ is bounded on when $0<p \leq 1$, and for $1<p<\infty$ the problem remained open. Recently $\mathrm{Li}\left[\mathrm{L}\right.$ gave a partial solution by proving the boundedness of $\mathcal{P}^{b, c}$ on $H^{p}$ for $p>1$ when $\operatorname{Re}(b+1)>\operatorname{Re} c \geq 1$. In this article we produce a different proof of that result.

Given a weight $\omega$ we define the function

$$
\psi(r)=\frac{1}{\omega(r)} \int_{r}^{1} \omega(u) d u \quad \text { for } 0 \leq r<1,
$$

and we call it the distortion function of $\omega$. We put $\psi(z)=\psi(|z|)$ for each $z \in \Delta$.

1.1. Definition. A weight $\omega$ is admissible if it satisfies the following conditions:

(i) There is a positive constant $A=A(\omega)$ such that

$$
\omega(r) \geq \frac{A}{1-r} \int_{r}^{1} \omega(u) d u \quad \text { for } 0 \leq r<1 .
$$

(ii) There is a positive constant $B=B(\omega)$ such that

$$
\omega^{\prime}(r) \leq \frac{B}{1-r} \omega(r) \quad \text { for } 0 \leq r<1 .
$$

(iii) For each sufficiently small positive $\delta$ there is a positive constant $C=C(\delta, \omega)$ such that

$$
\sup _{0 \leq r<1} \frac{\omega(r)}{\omega(r+\delta \psi(r))} \leq C .
$$

For details on admissible weights, see [Si4]. The following theorem was proved in [Si4].

1.2. TheOREM. Suppose $1 \leq p<\infty$ and $\omega$ is an admissible weight with distortion function $\psi$. Then

$$
\int_{\Delta}|f(z)|^{p} \omega(z) d m(z) \sim|f(0)|^{p}+\int_{\Delta}\left|f^{\prime}(z)\right|^{p} \psi(z)^{p} \omega(z) d m(z)
$$

for all $f \in \mathcal{H}$.

The notation $\sim$ means that there are finite positive constants $C$ and $C^{\prime}$ independent of $f$ (but possibly dependent on $p$ ) such that the left and right sides $L(f)$ and $R(f)$ satisfy

for all analytic $f$.

$$
C R(f) \leq L(f) \leq C^{\prime} R(f)
$$

1.3. EXAMPLE. A straightforward computation shows that the standard weight $\omega(r)=(1-r)^{\alpha}, \alpha>-1$, for $r \in(0,1)$ is admissible and its distortion function satisfies $\psi(r) \sim 1-r$. 
Henceforth $C$ denotes a positive constant whose value is different at different occurrences. The constant may depend on the parameters $a, p$ and we write in that case $C(a, p)$.

2. Boundedness of generalized Cesàro operators. In this section, we discuss the boundedness of generalized Cesàro operators on Dirichlet spaces $\mathcal{D}_{p, a}$ and Hardy spaces $H^{p}$.

$\mathcal{P}^{b, c}$ has an equivalent integral representation (see [AHLNP] which is given in the following lemma.

2.4. Lemma. For $b, c \in \mathbb{C}$ with $\operatorname{Re}(b+1)>\operatorname{Re} c>0$, we have

$$
\mathcal{P}^{b, c} f(z)=\frac{z^{-b}}{B} \int_{0}^{z} \zeta^{c-1}(z-\zeta)^{b-c} \frac{f(\zeta)}{(1-\zeta)^{b+1-c}} F(\zeta) d \zeta,
$$

where $F(\zeta)=F(c-1, c-b-1 ; c ; \zeta)$ and $B=B(c, b+1-c)$ is the usual beta function.

For each $t \in[0,1]$, we choose the path of integration between 0 and $z$ as

$$
\Gamma(t)=\phi_{t}(z)=\frac{t z}{1+(t-1) z} .
$$

Using this path in 2.5 , we have

$$
\begin{aligned}
& \mathcal{P}^{b, c} f(z) \\
& =\frac{z^{-b}}{B} \int_{0}^{1} \phi_{t}(z)^{c-1}\left(z-\phi_{t}(z)\right)^{b-c} \frac{f\left(\phi_{t}(z)\right)}{\left(1-\phi_{t}(z)\right)^{b+1-c}} F\left(\phi_{t}(z)\right) \phi_{t}^{\prime}(z) d t \\
& =\frac{1}{B} \int_{0}^{1} \frac{t^{c-1}(1-t)^{b-c}}{(1+(t-1) z)^{c}} f\left(\phi_{t}(z)\right) F\left(\phi_{t}(z)\right) d t .
\end{aligned}
$$

Define

$$
T_{t} f(z)=\omega_{t}^{c}(z) f\left(\phi_{t}(z)\right) F\left(\phi_{t}(z)\right) \quad \text { for } t \in(0,1],
$$

where $\omega_{t}(z)=t /(1+(t-1) z)$ and $F(z)=F(c-1, c-b-1 ; c ; z)$. Then

$$
\mathcal{P}^{b, c} f(z)=\frac{1}{B} \int_{0}^{1} \frac{1}{t} T_{t}(f(z))(1-t)^{b-c} d t .
$$

Now we recall the following result from [S1].

2.8. Lemma. Let $f \in \mathcal{D}_{p, a}, p>0, a>p-1$. Then there is a constant $C=C(a, p)$ such that

$$
|f(z)| \leq \frac{C}{(1-|z|)^{(a+2) / p-1}}\|f\|_{\mathcal{D}_{p, a}} .
$$


We will make use of this lemma to prove our next result which is given in the following theorem.

2.9. Theorem. Let $b, c \in \mathbb{C}$ be such that $\operatorname{Re}(b+1)>\operatorname{Re} c>0$. Suppose $a>p-1$ and $f \in \mathcal{D}_{p, a}$. If $p>1$ and $\operatorname{Re} c \geq 1$, then there are constants $C=C(a, p)$ and $\beta>0$ such that

$$
\left\|T_{t}\right\|_{\mathcal{D}_{p, a}} \leq C t^{\beta}\|f\|_{\mathcal{D}_{p, a}} .
$$

For $0<\operatorname{Re} c<1$, the above inequality is true if $p \geq 2$.

Proof. For the sake of simplicity, assume $c$ to be real and positive, since the proof for $c$ complex can be easily modified. Suppose $f \in \mathcal{D}_{p, a}$. Then using the definition of $T_{t}$ we have

$$
\begin{aligned}
\left\|T_{t}\right\|_{\mathcal{D}_{p, a}}^{p} & =\left|T_{t}(f(0))\right|^{p}+\int_{\Delta}\left|\left(\omega_{t}^{c}(z) f\left(\phi_{t}(z)\right) F\left(\phi_{t}(z)\right)\right)^{\prime}\right|^{p}(1-|z|)^{a} d m(z) \\
& \leq t^{c p}|f(0)|^{p}+c_{p}\left(I_{1}+I_{2}+I_{3}\right),
\end{aligned}
$$

where

$$
\begin{aligned}
& I_{1}=\int_{\Delta}\left|\left(\omega_{t}^{c}(z)\right)^{\prime}\right|^{p}\left|f\left(\phi_{t}(z)\right) F\left(\phi_{t}(z)\right)\right|^{p}(1-|z|)^{a} d m(z), \\
& I_{2}=\int_{\Delta}\left|\omega_{t}^{c}(z)\right|^{p}\left|f\left(\phi_{t}(z)\right)^{\prime}\right|^{p}\left|F\left(\phi_{t}(z)\right)\right|^{p}(1-|z|)^{a} d m(z), \\
& I_{3}=\int_{\Delta}\left|\omega_{t}^{c}(z)\right|^{p}\left|f\left(\phi_{t}(z)\right)\right|^{p}\left|F\left(\phi_{t}(z)\right)^{\prime}\right|^{p}(1-|z|)^{a} d m(z) .
\end{aligned}
$$

We have

$$
\left|\left(\omega_{t}^{c}(z)\right)^{\prime}\right|=\frac{c t^{c}(1-t)}{|1+(t-1) z|^{c+1}} \quad \text { and } \quad \frac{1}{1-\left|\phi_{t}(z)\right|} \leq \frac{|1+(t-1) z|}{1-|z|} .
$$

The boundedness of $F\left(\phi_{t}(z)\right)=F\left(c-1, c-b-1 ; c ; \phi_{t}(z)\right)$ follows from $\operatorname{Re}(b+1)>\operatorname{Re}(c-1)$, on $|z| \leq 1$. Since $F\left(\phi_{t}(z)\right)=F\left(c-1, c-b-1 ; c ; \phi_{t}(z)\right)$ is bounded, Lemma 2.8 and the above calculation shows that

$$
\begin{aligned}
& I_{1} \leq C\|f\|_{\mathcal{D}_{p, a}}^{p} \int_{\Delta}\left|\left(\omega_{t}^{c}(z)\right)^{\prime}\right|^{p} \frac{(1-|z|)^{a}}{\left(1-\left|\phi_{t}(z)\right|\right)^{a+2-p}} d m(z) \\
\leq & C\|f\|_{\mathcal{D}_{p, a}}^{p} c^{p} t^{c p}(1-t)^{p} \int_{\Delta} \frac{1}{|1+(t-1) z|^{(c+1) p}} \frac{(1-|z|)^{a}}{\left(1-\left|\phi_{t}(z)\right|\right)^{a+2-p}} d m(z) \\
\leq & C\|f\|_{\mathcal{D}_{p, a}}^{p} t^{c p}(1-t)^{p} \int_{\Delta} \frac{|1+(t-1) z|^{a+2-(c+2) p}}{(1-|z|)^{2-p}} d m(z) .
\end{aligned}
$$

For any $b>0, t \in[0,1]$ and $z \in \Delta$, we have

$$
\frac{1}{|1+(t-1) z|^{b}} \leq \frac{1}{(1-|z|)^{b}} \quad \text { and } \quad t^{b} \leq|1+(t-1) z|^{b} .
$$


Choose $\epsilon>0$ such that

$$
\epsilon< \begin{cases}\min \{1, p-1,(a-p+1) / 2\} & \text { if } c \geq 1, \\ \min \{1, p-1,(a-p+1) / 2, c p\} & \text { if } 0<c<1\end{cases}
$$

By (2.11), we obtain

$$
\begin{aligned}
& \frac{|1+(t-1) z|^{a+2-(c+2) p}}{(1-|z|)^{2-p}} \\
& \quad=\frac{|1+(t-1) z|^{a-p+1-2 \epsilon}}{|1+(t-1) z|^{c p-\epsilon}|1+(t-1) z|^{p-1-\epsilon}} \frac{(1-|z|)^{p-1-\epsilon}}{(1-|z|)^{1-\epsilon}} \\
& \quad \leq \frac{1}{(1-|z|)^{1-\epsilon} t^{c p-\epsilon}} .
\end{aligned}
$$

Using (2.12) in (2.10) it is easy to see that

$$
I_{1} \leq C\|f\|_{\mathcal{D}_{p, a}}^{p}(1-t)^{p} t^{\epsilon} .
$$

Now for $I_{2}$ we have

$$
\begin{aligned}
I_{2}= & \int_{\Delta}\left|\omega_{t}^{c}(z)\right|^{p}\left|f^{\prime}\left(\phi_{t}(z)\right)\right|^{p}\left|\left(\phi_{t}(z)\right)^{\prime}\right|^{p}\left|F\left(\phi_{t}(z)\right)\right|^{p}(1-|z|)^{a} d m(z) \\
= & \int_{\Delta}\left|\omega_{t}^{c}(z)\right|^{p}\left|f^{\prime}\left(\phi_{t}(z)\right)\right|^{p}\left|\left(\phi_{t}(z)\right)^{\prime}\right|^{p-2}\left|F\left(\phi_{t}(z)\right)\right|^{p}\left(\frac{1-|z|}{1-\left|\phi_{t}(z)\right|}\right)^{a} \\
& \times\left(1-\left|\phi_{t}(z)\right|\right)^{a} d m\left(\phi_{t}(z)\right) .
\end{aligned}
$$

Choose $\epsilon_{1}>0$ such that

$$
\epsilon_{1}< \begin{cases}\min \{a-p+2,2 p-2\} & \text { if } c \geq 1, \\ \min \{a-p+2,(c+1) p-2\} & \text { if } 0<c<1 .\end{cases}
$$

One can quickly obtain the following:

$$
\begin{aligned}
& \left|\omega_{t}^{c}(z)\right|^{p}\left|\left(\phi_{t}(z)\right)^{\prime}\right|^{p-2}\left(\frac{1-|z|}{1-\left|\phi_{t}(z)\right|}\right)^{a} \leq t^{(c+1) p-2} \frac{|1+(t-1) z|^{a-p+2-\epsilon_{1}}}{|1+(t-1) z|^{(c+1) p-2-\epsilon_{1}}} \\
& \leq t^{\epsilon_{1}} \quad \text { (using (2.11)), }
\end{aligned}
$$

therefore we have

$$
I_{2} \leq C\|f\|_{\mathcal{D}_{p, a}}^{p} t^{\epsilon_{1}}
$$

Further we have

$$
F^{\prime}\left(\phi_{t}(z)\right)=\frac{(c-1)(c-b-1)}{c} F\left(c, c-b ; c+1 ; \phi_{t}(z)\right)\left(\phi_{t}(z)\right)^{\prime},
$$

and since $F\left(c, c-b ; c+1 ; \phi_{t}(z)\right)$ is bounded for $\operatorname{Re}(b+1)>\operatorname{Re} c$ on $|z| \leq 1$, 
we have

$$
\begin{aligned}
I_{3} & \leq C \int_{\Delta}\left|\omega_{t}^{c}(z)\right|^{p}\left|f\left(\phi_{t}(z)\right)\right|^{p}\left|\left(\phi_{t}(z)\right)^{\prime}\right|^{p}(1-|z|)^{a} d m(z) \\
& =C \int_{\Delta} \frac{t^{(c+1) p}}{|1+(t-1) z|^{(c+2) p}}\left|f\left(\phi_{t}(z)\right)\right|^{p}(1-|z|)^{a} d m(z) \\
& \leq C\|f\|_{\mathcal{D}_{p, a}}^{p} t^{(c+1) p} \int_{\Delta} \frac{1}{|1+(t-1) z|^{(c+2) p}} \frac{(1-|z|)^{a}}{\left(1-\left|\phi_{t}(z)\right|\right)^{a+2-p}} d m(z) \\
& \leq C\|f\|_{\mathcal{D}_{p, a}^{p}}^{p} t^{(c+1) p} \int_{\Delta} \frac{|1+(t-1) z|^{a+2-(c+3) p}}{(1-|z|)^{2-p}} d m(z) \\
& \left.\leq C\|f\|_{\mathcal{D}_{p, a}^{p}}^{p} t^{(c+1) p} \int_{\Delta} \frac{1}{(1-|z|)^{1-\epsilon} t^{(c+1) p-\epsilon}} d m(z) \quad \text { (using (2.11) }\right)
\end{aligned}
$$

by choosing the same $\epsilon$ as in $I_{1}$, the last inequality shows

$$
I_{3}<C\|f\|_{\mathcal{D}_{p, a}}^{p} t^{\epsilon} .
$$

Finally, combining all the above results for $I_{1}, I_{2}$ and $I_{3}$, we obtain

$$
\begin{aligned}
\left\|T_{t}\right\|_{\mathcal{D}_{p, a}}^{p} & \leq t^{c p}|(f(0))|^{p}+c_{p} C\left((1-t)^{p} t^{\epsilon}\|f\|_{\mathcal{D}_{p, a}}^{p}+t^{\epsilon 1}\|f\|_{\mathcal{D}_{p, a}}^{p}+t^{\epsilon}\|f\|_{\mathcal{D}_{p, a}}^{p}\right) \\
& \leq C t^{c p}\|f\|_{\mathcal{D}_{p, a}}^{p}+c_{p} C\|f\|_{\mathcal{D}_{p, a}}^{p}\left(t^{\epsilon}+t^{\epsilon}+t^{\epsilon}\right) \quad(\text { Lemma 2.8) } \\
& \leq C t^{\beta}\|f\|_{\mathcal{D}_{p, a}}^{p}
\end{aligned}
$$

for $\beta=\min \left\{\epsilon, \epsilon_{1}\right\}>0$ and $C=\max \left(C, c_{p} C\right)$, completing the proof.

Now we are in a position to prove our next result which concerns the boundedness of generalized Cesàro operators on Dirichlet spaces.

2.13. Theorem. Let $b, c \in \mathbb{C}$ be such that $\operatorname{Re}(b+1)>\operatorname{Re} c>0$. Suppose $a>p-1$. Then the generalized Cesàro operator $\mathcal{P}^{b, c}$ is bounded on $\mathcal{D}_{p, a}$ when $p>1$ and $\operatorname{Re} c \geq 1$. Further, $\mathcal{P}^{b, c}$ is bounded on $\mathcal{D}_{p, a}$ for $0<\operatorname{Re} c<1$ if $p \geq 2$.

Proof. We give the proof only for $b, c$ real with $b+1>c>0$. For the proof of the complex case, we just nead to note the following, for $t \in(0,1)$ :

$$
\left|t^{c-1}\right|=t^{\operatorname{Re} c-1}, \quad\left|(1-t)^{b-c}\right|=(1-t)^{\operatorname{Re}(b-c)}
$$

and

$$
\left|(1-z t)^{b+1-c}\right|=|1-t z|^{\operatorname{Re}(b+1-c)} e^{-\operatorname{Im}(b+1-c) \arg (1-t z)} .
$$

Here we choose the principal argument for $\arg (1-t z)$ such that $\arg (1-t z)=0$ at $z=0$, and we note that $|\arg (1-t z)|<\pi / 2$ for $z \in \Delta$. Moreover, the integral $\int_{0}^{1} t^{c-1}(1-t)^{b-c} d t$ converges since by the hypotheses $\operatorname{Re}(b+1-c)>0$ 
and $\operatorname{Re} c>0$, and therefore it suffices to assume $b$ and $c$ are real, and that $b+1>c>0$ in the proof.

To prove the theorem it is sufficient to show that

$$
\left\|\mathcal{P}^{b, c} f\right\|_{\mathcal{D}_{p, a}}^{p} \leq C\|f\|_{\mathcal{D}_{p, a}}^{p}
$$

for some $C>0$, depending on $b, c, p$ and $a$. Using the integral representation given by (2.7), we have

$\left\|\mathcal{P}^{b, c} f\right\|_{\mathcal{D}_{p, a}}^{p}$

$$
\begin{aligned}
& =\left|\mathcal{P}^{b, c} f(0)\right|^{p}+\frac{1}{B} \int_{\Delta}\left|\int_{0}^{1} t^{-1}(1-t)^{b-c} T_{t}^{\prime}(f(z)) d t\right|^{p}(1-|z|)^{a} d m(z) \\
& \leq\left|\mathcal{P}^{b, c} f(0)\right|^{p}+\frac{1}{B} \int_{\Delta}\left(\int_{0}^{1} t^{-1}(1-t)^{b-c}\left|T_{t}^{\prime}(f(z))\right| d t\right)^{p}(1-|z|)^{a} d m(z) \\
& \leq|f(0)|^{p}+\frac{1}{B^{p}} \int_{0}^{1}\left(\frac{1}{t}\left(\int_{\Delta}\left|T_{t}^{\prime}(f(z))\right|^{p}(1-|z|)^{a} d m(z)\right)^{1 / p}(1-t)^{b-c} d t\right)^{p}
\end{aligned}
$$

(Minkowski inequality)

$$
\begin{aligned}
& \leq|f(0)|^{p}+\left(\frac{1}{B^{p}} \int_{0}^{1} t^{-1}(1-t)^{b-c}\left\|T_{t}\right\|_{\mathcal{D}_{p, a}} d t\right)^{p} \\
& \leq|f(0)|^{p}+\frac{C^{p}}{B^{p}}\|f\|_{\mathcal{D}_{p, a}}^{p}\left(\int_{0}^{1} t^{\beta-1}(1-t)^{b-c} d t\right)^{p} \quad \text { (Theorem 2.9) } \\
& \leq C\|f\|_{\mathcal{D}_{p, a}}^{p}+\frac{C^{p}}{B^{p}} B^{p}(\beta, b-c+1)\|f\|_{\mathcal{D}_{p, a}}^{p} \quad \text { (Lemma 2.8) } \\
& \leq C\|f\|_{\mathcal{D}_{p, a}}^{p},
\end{aligned}
$$

where $C=\max \left(C,\left(C^{p} / B^{p}\right) B^{p}(\beta, b-c+1)\right)$, which completes the proof.

2.14. Theorem. Let $b, c \in \mathbb{C}$ be such that $\operatorname{Re}(b+1)>\operatorname{Re} c>0$. Then for $\alpha>-1$, the generalized Cesàro operator $\mathcal{P}^{b, c}$ is bounded on $\mathcal{B}_{\left(1-|z|^{2}\right)^{\alpha}}^{p}$ when $p>1$ and $\operatorname{Re} c \geq 1$, and also for $0<\operatorname{Re} c<1$ when $p \geq 2$.

Proof. Suppose $f \in \mathcal{B}_{\left(1-|z|^{2}\right)^{\alpha}}^{p}$ and $p>1$. Since $\mathcal{P}^{b, c} f(0)=f(0)$, using the previous theorem we have

$$
\int_{\Delta}\left|\left(\mathcal{P}^{b, c}\right)^{\prime} f(z)\right|^{p}(1-|z|)^{a} d m(z) \leq C \int_{\Delta}\left|f^{\prime}(z)\right|^{p}(1-|z|)^{a} d m(z) .
$$

Let $\omega(r)=(1-r)^{\alpha}, \alpha>-1$. If we take $a=\alpha+p$, as $\psi(r) \sim 1-r$ is the distortion function for $\omega(r)$, the theorem follows from the above inequality and Theorem 1.2 , 
We state the boundedness of the operators $\mathcal{P}^{b, c}$ on Hardy spaces. We recall the following result from [GS] which we will use to prove our next theorem.

2.15. Lemma. For the Hardy space norms of the weighted composition operators

$$
U_{t} f(z)=\frac{1}{1+(t-1) z} f\left(\frac{t z}{1+(t-1) z}\right)
$$

we have:

(i) If $2 \leq p<\infty$ then

$$
\left\|U_{t}\right\| \leq t^{-1+1 / p}, \quad 0<t \leq 1 .
$$

(ii) If $1<p<2$ then there is a constant $C_{p}$ depending only on $p$ such that

$$
\left\|U_{t}\right\| \leq C_{p} t^{-1+1 / p}, \quad 0<t \leq 1 .
$$

2.16. Theorem. Let $b, c \in \mathbb{C}$ be such that $\operatorname{Re}(b+1)>\operatorname{Re} c \geq 1$. The generalized Cesàro operator $\mathcal{P}^{b, c}$ is bounded on $H^{p}$ for $1<p<\infty$.

Proof. Let $f \in H^{p}, 1<p<\infty$. Our aim is to show that

$$
M_{p}\left(r, \mathcal{P}^{b, c} f\right) \leq C\|f\|_{p}
$$

for some $C>0$. Suppose $b, c \in \mathbb{C}$ with $\operatorname{Re}(b+1)>\operatorname{Re} c>0$. It is sufficient to prove the assertion for real $b$ and $c$, because of the same reasoning as in the previous theorem. For each $t \in(0,1]$, the function $\phi_{t}$ given by

$$
\phi_{t}(z)=\frac{t z}{1+(t-1) z}
$$

maps the disc into itself. Then (see [D, p. 29]) $f\left(\phi_{t}(z)\right) \in H^{p}$ for $z \in \Delta$. Since $F(c-1, c-b-1, c ; z)$ is bounded for $b+1>c-1$, we see that $F\left(\phi_{t}(z)\right)=F\left(c-1, c-b-1, c ; \phi_{t}(z)\right)$ is bounded on $|z| \leq 1$ and therefore $F\left(\phi_{t}(z)\right) f\left(\phi_{t}(z)\right)$ is bounded on $H^{p}$ for $1<p<\infty$.

We can easily obtain (see for example [GS, p. 4]) that for $c>0$, the weight functions $\varpi_{t}(z)=1 /(1+(t-1) z)^{c}$ are bounded on $\Delta$ for each $t \in(0,1]$. Thus, for each $t \in(0,1]$ the weighted composition operators $S_{t}$ defined by

$$
S_{t} f(z)=\varpi_{t}(z) f\left(\phi_{t}(z)\right) F\left(\phi_{t}(z)\right)
$$

are bounded on $H^{p}$. Since $c \geq 1, F(c-1, c-b-1, c ; z)$ is bounded for 
$b+1>c-1$ and using the second inequality of (2.11), we find

$$
\begin{aligned}
\left\|S_{t}(f)\right\|_{p} & =\lim _{r \rightarrow 1}\left\{\frac{1}{2 \pi} \int_{0}^{2 \pi}\left|S_{t}\left(f\left(r e^{i \theta}\right)\right)\right|^{p} d \theta\right\}^{1 / p} \\
& =\lim _{r \rightarrow 1}\left\{\frac{1}{2 \pi} \int_{0}^{2 \pi}\left|\frac{1}{1+(t-1) z}\right|^{(c-1) p}\left|F\left(\phi_{t}\left(r e^{i \theta}\right)\right)\right|^{p}\left|U_{t} f\left(r e^{i \theta}\right)\right|^{p} d \theta\right\}^{1 / p} \\
& \leq C t^{1-c}\left\|U_{t}(f)\right\|_{p} .
\end{aligned}
$$

Using Lemma 2.15 in the above inequality, we have

$$
\left\|S_{t}\right\|_{p} \leq \begin{cases}t^{-c+1 / p} & \text { if } 2 \leq p<\infty \\ C C_{p} t^{-c+1 / p} & \text { if } 1<p<2\end{cases}
$$

Now $\mathcal{P}^{b, c}$ defined in 2.6 can be written in the following form:

$$
\mathcal{P}^{b, c} f(z)=\frac{1}{B} \int_{0}^{1} S_{t} f(z) t^{c-1}(1-t)^{b-c} d t .
$$

Using Minkowski's inequality and the boundedness of $S_{t}$ on $H^{p}$, we have

$$
\begin{aligned}
M_{p}\left(r, \mathcal{P}^{b, c} f\right) & \leq \frac{1}{B}\left(\frac{1}{2 \pi} \int_{0}^{2 \pi}\left(\int_{0}^{1}\left|S_{t}\left(f\left(r e^{i \theta}\right)\right)\right| t^{c-1}(1-t)^{b-c} d t\right)^{p} d \theta\right)^{1 / p} \\
& \leq \frac{1}{B} \int_{0}^{1}\left(\frac{1}{2 \pi} \int_{0}^{2 \pi}\left|S_{t}\left(f\left(r e^{i \theta}\right)\right)\right|^{p} d \theta\right)^{1 / p} t^{c-1}(1-t)^{b-c} d t \\
& \leq \frac{C}{B}\|f\|_{p} \int_{0}^{1} t^{1 / p-1}(1-t)^{b-c} d t=C\|f\|_{p},
\end{aligned}
$$

which completes the proof.

Acknowledgments. The author wishes to acknowledge the financial support as a post doctoral fellow from NBHM (National Board of Higher Mathematics, India) (No. 40/11/2004-R\&D-II/5605).

\section{References}

[AHLNP] M. R. Agrawal, P. G. Howlett, S. K. Lucas, S. Naik and S. Ponnusamy, Boundedness of generalized Cesàro averaging operator on certain function spaces, J. Comput. Appl. Math. 126 (1998), 3553-3560.

[AAR] G. E. Andrews, R. Askey and R. Roy, Special Functions, Cambridge Univ. Press, 1999.

[CLS] D. C. Chang, S. Li and S. Stević, On some integral operators on the unit polydisk and the unit ball, Taiwanese J. Math. 11 (2007), 1251-1286.

[CS1] D. C. Chang and S. Stević, The generalized Cesàro operator on the unit polydisk, ibid. 7 (2003), 293-308. 
[CS2] D. C. Chang and S. Stević, A note on weighted Bergman spaces and Cesàro operator, Nagoya Math. J. 180 (2005), 77-99.

[D] P. L. Duren, Theory of $H^{p}$ Spaces, Academic Press, New York, 1970.

[G] P. Galanopoulos, The Cesàro operator on Dirichlet spaces, Acta Sci. Math. (Szeged) 67 (2001), 411-420.

[GS] P. Galanopoulos and A. G. Siskakis, Hausdorff matrices and composition operators, Illinois J. Math. 45 (2001), 757-773.

[L] S. Li, A note on boundedness of generalized Cesàro operators on certain function spaces, Indian J. Math. 48 (2006), 103-111.

[M] J. Miao, The Cesàro operator is bounded on $H^{p}$ for $0<p<1$, Proc. Amer. Math. Soc. 116 (1992), 1077-1079.

[Si1] A. G. Siskakis, Composition semigroups and the Cesàro operator on $H^{p}$, J. London Math. Soc. 36 (1987), 153-164.

[Si2] - The Cesàro operator is bounded on $H^{1}$, Proc. Amer. Math. Soc. 110 (1990), 461-462.

[Si3] - On the Bergman space norm of the Cesàro operator, Arch. Math. (Basel) 67 (1996), 312-318.

[Si4] - Weighted integral of analytic funcions, Acta Sci. Math. (Szeged) 66 (2000), 651-664.

[St] K. Stempak, Cesàro averaging operators, Proc. Roy. Soc. Edinburgh Sect. A 124 (1994), 121-126.

[S1] S. Stević, The generalized Cesàro operator on Dirichlet spaces, Studia Sci. Math. Hungar. 40 (2003), 83-94.

[S2] - Cesàro averaging operators, Math. Nachr. 248-249 (2003), 185-189.

[T] N. M. Temme, Special Functions: An Introduction to the Classical Functions of Mathematical Physics, Wiley, New York, 1996.

[X] J. Xiao, Cesàro type operators on Hardy, BMOA and Bloch spaces, Arch. Math. (Basel) 68 (1997), 398-406.

Sunanda Naik

Institute of Mathematics and Applications

Andharua

Bhubaneswar 751 003, India

E-mail: spn20@yahoo.com

Received 15.7.2009

and in final form 27.8.2009 
